\title{
Reuse of Waste from the Perspective of Circular Economy
}

\author{
Liu Yijie ${ }^{1, a^{*}}$ and Zhang Shu ${ }^{1, b}$ \\ ${ }^{1}$ Wuhan University of Science and Technology, Art Design College Wuhan ,Hubei, China \\ a308864991@qq.com; ${ }^{b}$ 3609599@qq.com
}

Keywords: Circular Economy; Industrial Waste; Reuse

\begin{abstract}
In the process of urbanization in China, the scale of the city has expanded, and some factories that are harmful are also moving from the center of the city. Therefore, many industrial wasteland, old factories and some waste industrial equipment have been left in the city. Brought a series of social and environmental issues. The emergence and development of industrial landscapes have always been closely related to the development of cities. It is precisely because of the continuous demand and demand for natural resources, especially non-renewable resources, the depletion of resources has caused a large number of abandoned mine sites. How to put these Reuse of land and waste to solve pollution problems.

With the increasing industrialization of the city, industrialization has also promoted urbanization and created many urban problems. The urban industrial wasteland has also caused many urban industrial landscape reconstruction projects. According to the different objects and scales, it can be roughly divided into the protection and reuse of industrial heritage, post-industrial landscape design, and creative industrial park mode. Many of the disciplines involved include heritage protection, landscape design, urban planning and design. Architectural design and environmental art design. Further, the post-industrial landscape includes post-industrial parks, communities, plazas, communities, etc. For example, the Shanghai World Expo Park is a typical post-industrial park, and the urban best practice area in the Expo Park is a typical post-industrial. Square, Canada's Granville Island is one of the most successful post-industrial art communities, and Tianjin Vanke's Crystal City is also a representative of the post-industrial community. Finally, the result of industrialization and urbanization is the increasing production of industrial waste and urban domestic waste. The city is gradually surrounded by garbage dumps. The formerly remote landfills are getting closer to the city center, opening up new ones. Before the waste treatment plant, the landscape treatment of these special plots was imminent. The most successful example in this regard is Fresh Kill Landfill in New York, USA. The world's largest landfill is being transformed into a new ecological park.
\end{abstract}

\section{Recycling of construction waste}

Taking the surrounding land of WISCO in Qingshan as an example, every ton of scrap steel used in the construction of waste steel can produce $850 \mathrm{~kg}$ of good steel, save 1.7 tons of iron ore, 0.68 tons of coke or 1 ton of marked coal, and $280 \mathrm{~kg}$ of limestone., less than 20 tons of various ore, less transport 30 tons. Compared with the raw material steelmaking, the air pollution is reduced by $86 \%$, the harmful dust is reduced by $16 \mathrm{~kg} / \mathrm{ton}$, the solid waste is reduced by $12 \%$, the harmful substances are reduced by $1 / 3$, and the water pollution is reduced by $76 \%$, saving the natural Consumption also solves the problem of environmental pollution and land occupation caused by the natural decomposition process of construction and industrial waste in the ecosystem.

\section{Landscape Leveling and Integration}

On the one hand, the landscape greening layer is the basic greening demand, and the greening planting has the universal significance of the general site greening function. However, the project site is located in the heavy industrial area of Wuhan City. The industrial pollution is very serious, and the greening can reduce the pollution to a certain extent. On the other hand, the tree array and three-dimensional greening in the green square, focusing on the visual needs under the premise of 
meeting the ecological benefits, designing the landscape of the tree array combined with the light and spray, and the three-dimensional greening on the landscape platform not only increases the greening. The area has also beautified the landscape.

In the design of the landscape, the principle of participation and practicality is dominant. The places where people pass most often, the places where people flow are most dense and dense, provide small landscapes and seats, and some people have places to have landscapes. On the one hand, the essay can be combined with functions and on the other hand to meet the needs of the vision. The landscape of the landscape platform mainly focuses on observability and fun.

Pavement layer The pavement layer is mainly divided into two levels: the ground pavement of the Green Plaza Square, which is mainly made of cement floor covering, which is in line with the characteristics of the landscape. Second, the pavement of the viewing platform is made of scrap steel with industrial characteristics, which makes the entrance prospect and the back platform style unified and integrated.

Protection of the original industrial site

The overall layout of the skeleton structure and its spatial nodes, constituent elements, etc. are fully protected, not just partially retained. On the premise of structural analysis of various independent industrial facilities such as steelmaking blast furnaces, gas storage tanks, workshops, ore silos, the overall spatial scale and landscape features of the old plant area are formed in the green grass landscape park on the project side road side. The framework is preserved and extended. Through the comprehensive utilization of various industrial facilities on the site, the Greenland Landscape Park can accommodate various activities such as visits, information consultation, catering, sports, gatherings, performances, leisure, entertainment, etc., fully demonstrating the specific implementation of the design. Technical reality and economic feasibility.

\section{Water Pollution Purification and Rainwater Harvesting}

In the design, the Castle Peak Port channel, which was originally used as a public open space drain, was treated to separate the sewage from the water purification system. The length of the green area of Qingshangang River is about $1.5 \mathrm{~km}$. In the past, domestic sewage, industrial wastewater, rainwater, garbage, etc. will be discharged into the river, making the water pollution serious and dirty. The smell of summer is bad and affects air quality. After design and transformation, all kinds of sewage will be discharged from the underground sewage pipe, and the purified water will be in the form of a canal to avoid direct contact between the water and the contaminated soil; collecting rainwater will enter the cooling pool and clear the sewage through the pipeline. After the sedimentation tank of the mud is filtered, it enters the wetland of the canal for further purification.

This is a transformation of the ecological environment combined with design, more emphasis on the importance of ecological impact design, aimed at the natural circulation of the process to properly guide the environmental improvement of the water body.

\section{Wind Energy Utilization}

In ancient times, there was a "wind power". The "wind tower" planned to be placed on the shore of Qingshan Port can use the wind to raise the purified water from the bottom of the river to the upper level, which can be used as irrigation water for the dry season; in other seasons, The elevated water can be recharged into the river. Through this water energy cycle process, on the one hand, it creates an interesting and interesting water flow and a falling audio-visual environment in the sensory environment; on the other hand, it increases the contact between water and oxygen through the flow of water, and improves the purification of water. Quality has improved the quality of the surrounding environment. 


\section{Vegetation protection}

Vegetation with its own purification function, targeted selection of vegetation required by the site, preferred functionality, followed by aesthetics, paying attention to landscape aesthetics on the basis of meeting the required functions of the site vegetation. First, the original site vegetation is retained, because it is abandoned. Wild vegetation that is able to carry out ecological succession on the contaminated soil is a rare experimental and research object of ecologists and should be protected. On the basis of protecting the original site vegetation, the secondary design of plant design is completed, and the concept of protecting the original ecological environment is extended to extend the concept of "ecological barrier".

\section{Industrial waste utilization}

The riverbed of roads, plazas and new clean water canals is laid by waste slag deposited in the factory area formed by industrial production. As well as various landscape structures and landscape pieces in the design, it can not only form the conceptual elements of the entire landscape design, but also penetrate the spirit of design. For example, in the design of the tree pool transformed with waste containers, not only the reuse of waste materials, but also the interesting interest of the landscape in the original design elements, that is, the original concept of the site.

\section{Disposal of abandoned industrial buildings, structures and industrial facilities}

Landscape design is based on the industrial landscape, the design of the original industrial landscape on the site treatment, is an important part of the design, but also a part of the foundation, but also retain the original site habits part. The industrial landscape refers to abandoned industrial buildings, structures, machinery and facilities related to industrial production.

In the design, three large ways are adopted to preserve or reproduce the industrial landscape on the site, and the whole is retained. As the name suggests, the overall reservation is to inherit the original state of the factory, including the industrial buildings, structures and equipment facilities and the road system and functional division of the factory. In the reconstructed park, the operational process of the previous industrial production can be perceived. This method is the most intuitive reproduction of the original venue function, but also respects the atmosphere of the original venue. The second is partial retention. That is to say, leaving a fragment of the abandoned industrial landscape or part of the point-like memory, making it a landmark and nodular landscape of the park. The retained fragments may be industrial landscapes of typical origin, representing the original factory character, industrial buildings of historical value, or old buildings of good quality and only need to be properly repaired and reinforced, using appropriate modern The design technique reproduces its charm. The third is the component retention. That is, retaining a part of a structure, building, facility structure or structure, such as walls, foundations, frames, trusses and other components. From these components, we can basically see the clues of the previous industrial landscape, causing people's association and memory. This way is more to create an atmosphere in the spirit and create an environment. The abandoned industrial building structures or facilities retained after the design can be used as sculptures on the site and can be used as visual highlights of the site landscape. We only emphasize visually iconic effects and do not give them a function. However, in most cases, abandoned factory facilities, after repairs and secondary design, can be reused and given new spiritual significance. For their secondary use I have summarized the following approaches:

Utilize the physical structure of the industrial landscape. The structure of the industrial landscape itself can easily be transformed into the structure of the park. The transport road and the original road system are linear systems that are linked to the various production nodes of the factory. It is easy to be transformed into a walkway system throughout the whole park through reservation and appropriate changes; the industrial blast furnace is slightly treated to be a good climb climbing facility and landscape culture sculpture. Nodes; and the storage bins surrounded by four sides can 
be arranged into special small gardens; the original site vegetation can be arranged to echo, the column frame of the building can be used as a support for climbing plants; the building foundation can be used as a reservoir Place a landscape sculpture in it.

Transformation of industrial buildings. The original industrial buildings in the site can be transformed into loft concert halls, theaters, museums, exhibition halls, hotels, offices and other entertainment or cultural buildings on the basis of the reserved space form. These old buildings will present their new and unique functionality in new ways, while giving the new building its preserved atmosphere and spiritual continuity.

Reuse old industrial equipment. Some equipment in the original factory of the site can be reused. We can play a role in the design and bring new features to the old industrial equipment that can be used.

Sometimes, due to conditional constraints or design requirements, some structures or facilities on industrial wasteland need to be demolished, and those components or industrial symbols that are removed can be reassembled and they will be formed in a new way through design. New architecture, sculpture and other landscapes.

In this landscape design, after reviewing the landscape design of many related industrial transformations, using the colorful modern art transformation techniques, it provides new ideas and new methods for dealing with industrial scenes on industrial waste land. Industrial building structures and facilities machinery can Becoming a creative material is not only a "waste update" but also a "cultural continuation", and industrial symbols can also become the theme language and loud clarification of artistic creation.

Some of the symbols boldly apply vivid colors to emphasize the soul of the industrial landscape, making it stand out, eye-catching and magnifying, turning the dilapidated industrial site into a beautiful humanity venue. On the one hand, the disorderly industrial landscape on industrial wasteland can be unified by the color of the same color. On the other hand, according to the psychological characteristics of people, color treatment can be used to express the specific theme of the landscape, and the visual impact can be enhanced in the psychological feeling. In industrial wasteland retrofits, colors can be used to indicate different zones and functional zones. In the process of symbol reproduction, some industrial components can bring about the effects of novel tension through dramatic and interesting treatments such as distortion, deformation, collision, mutation, bulging, collapse, fracture, and historical scene reproduction.

\section{Conclusion}

The landscape of the roadside green space is one of the elements of the green space structure system in this area, with the theme of open space integration and ecological restoration and reconstruction.

The construction of industrial landscape is a set of economic, environmental and social problems arising from the decline of industrial civilization in some developed countries, and the overall countermeasures of the system from the multi-dimensional perspectives and levels of ecology, culture and landscape, and in many aspects. Good results achieved through joint efforts. However, some scholars have proposed from the perspective of sustainable land development that the post-construction industrial landscape park is a transitional countermeasure for land use in industrial wasteland. After further ecological restoration and reconstruction, the land landscape will eventually return to industrial civilization. The idyllic state of the environment. It can be seen that this pre-judgment on the development of the land landscape pattern is full of ecological ideals, but it ignores the sufficient consideration and attention to the landscape significance of the industrial heritage carrying the cultural heritage mission. The future model of land use in industrial wasteland will depend on the superposition of multiple factors, and ecological factors are extremely important, but not all. Applying the ecological niche theory to further thinking, it can be seen that industrial sites and industrial landscapes in the industrial civilization period are the materialized representation of industrial production and the carrier driving social economic development, and are the symbol of regional economic glory. If it is compared to an ecological element, it is in line with the spatial and functional requirements of the real niche. However, when the structural crisis 
occurred, the industrial site gradually turned into an industrial wasteland, and the industrial landscape also evolved into an abandoned industrial facility, indicating that its actual state has not been consistent with the original niche; and the industrial landscape park was renewed as an industrial wasteland. A successful strategy is to find out the potential niche in line with the new environmental conditions, occupying and making full use of resources to transform the potential niche into a new real niche.

At present, the problem of regeneration of industrial wasteland is a problem that must be faced and solved by a large number of resource-based cities and old industrial bases in China and the rest of the world. The green space landscape planning and design of the Qingshan Park section of Wuhan Heping Avenue will serve as a classic example of the post-industrial landscape park. It has important reference significance for the practical exploration and theoretical research on the transformation and utilization of domestic industrial wasteland. The success of this case is based on its specific stage of social development, technology and economic background. For industrial wasteland under the current conditions of China, whether the industrial landscape green space is suitable for construction, what design method should be adopted and implementation Process and operation models, etc., still need to be carefully and scientifically analyzed and verified based on objective analysis and research.

\section{Acknowledgements}

This paper is supported by the subject Humanities and Social Sciences of the Department of industrial solid waste in landscape architecture by $16 \mathrm{Y} 020$.

\section{References}

[1] https://www.xzbu.com/2/view-3712333.htm

[2] Jakob Schneider.This Is Service Design Thinking. [M]. Wiley,2012

[3] $\mathrm{Pu}$ Dexiang, Fu Hongchun. Reinterpretation of Economics: Based on the Perspective of Happiness Economics [J]. Economist, 2013

[4] Gao Wei, Chen Gang. Integration and Expression of Cultural Landscape in Industrial Heritage Reconstruction[J]. China Urban Forestry, 2010

[5]Weilacher,U.Syntax of Landscape Architecture of Peter Latz and Partners,Birkh user,Basel.Boston.Berlin, 2008

[6]Qian Jing. Ecological Restoration and Landscape Regeneration of Industrial Wasteland[J].Jiangsu Architecture, 2003

[7] Luo hua. Ecological civilization--give prominence to the recycling of construction waste in landscaping $[\mathrm{J}]$ Garden, 2008

[8] Chen quanyuan, Liu huanhuan. The process of transferring the waste of steel industry to resources[J] Mining Metallurgical Engineering. 2007

[9] Wang qi. The treatment and recycling of industrial waste[M] Beijing: China Environmental Science Press, 2006

[10] Liang xiuyong. The recycling of construction waste[J]. Anhui technology, 2007 